Canad. Math. Bull. Vol. 22 (2), 1979

\title{
ON THE ZEROS OF POWER SERIES WITH LOGARITHMIC COEFFICIENTS
}

\author{
BY \\ U. STADTMÜLLER
}

0. Equivalence problems in Riesz-summability lead to the problem of finding the zeros of special power series in the unit circle, i.e.

$$
f_{\kappa}(z)=\sum_{n=0}^{\infty}(n+1)^{\kappa} z^{n} \quad \text { and } \quad g_{\kappa}(z)=\sum_{n=0}^{\infty}\left(1-c^{n+1}\right)^{\kappa} z^{n} \quad(0<c<1, \kappa>0) \text {. }
$$

These functions were investigated by Kuttner, Miesner, Peyerimhoff, Wirsing and others. Peyerimhoff [5] obtained the exact number of zeros of $f_{\kappa}(z)$ and $g_{\kappa}(z)$ from results on functions of the more general type

$$
f(z)=\sum_{n=0}^{k-1} b_{n} z^{n}+z^{k} \int_{0}^{1} \frac{d g(t)}{1-z t}
$$

$k \in \mathbb{N}_{0}, b_{n} \in \mathbb{R}$ for $n=0,1, \ldots, k-1, g(t) \nearrow$ on

$(0,1)$ and $z \in \mathbb{C}^{*}=\{x+i y \mid y \neq 0$ if $x \geq 1\}$.

Functions of this type are MacLaurin series with totally monotone coefficients.

Gawronski and Peyerimhoff [4] generalized the results of [5]. An interesting question in this connection is, how the zeros depend on the parameters, like $\kappa$ by $f_{\kappa}(z)$ or $\kappa$ and $c$ by $g_{\kappa}(z)$. Borwein and Kratz [1] proved that the zeros of $g_{\kappa}(z)$ are strictly increasing functions of $c$.

Wirsing [8] showed that the zeros of $f_{\kappa}(z)$ and $g_{\kappa}(z)$ are monotone in $\kappa$. The proof requires information on the zeros of the functions

$$
\frac{\partial}{\partial \kappa} f_{\kappa}(z)=\sum_{n=0}^{\infty}(n+1)^{\kappa} \log (n+1) z^{n}
$$

In this paper we will investigate the zeros of all derivatives of $f_{\kappa}(z)$, i.e. the functions

$$
f_{\kappa, \lambda}(z):=\left\{\begin{array}{lll}
\sum_{n=0}^{\infty}(n+1)^{\kappa} \log ^{\lambda}(n+1) z^{n} & \lambda \geq 0 \\
\sum_{n=1}^{\infty}(n+1)^{\kappa} \log ^{\lambda}(n+1) z^{n} & \lambda<0 &
\end{array} \quad \kappa \in \mathbb{R}\right.
$$

Like $f_{\kappa}(z)$ these functions admit analytic extension onto $\mathbb{C}^{*}$, and the analytic

Received by the editors January 4, 1978. 
extension will also be denoted by $f_{\kappa, \lambda}(z)$. The special case $\lambda=1$ was treated in [3], where power series with negative zeros are investigated.

In the first two sections of this paper we give a special form of the analytic continuation of $f_{\kappa, \lambda}(z)$ onto $\mathbb{C}^{*}$, which allows to use theorem 1 of [4] to obtain upper bounds for the number of zeros. We find the following results: $(k, m \in$ $\left.\mathbb{N}_{0}\right)$

\begin{tabular}{c|c|c}
\multicolumn{1}{c|}{$\kappa$} & $\lambda$ & $\begin{array}{c}\text { upper bounds for the number of zeros of } \\
f_{\kappa, \lambda}(z) \text { in } \mathbb{C}^{*}\end{array}$ \\
\hline $\begin{array}{c}\kappa \leq 0 \\
\kappa \leq 0\end{array}$ & $\begin{array}{c}\lambda<0 \\
m<\lambda \leq m+1\end{array}$ & 1 \\
$k<\kappa \leq k+1$ & $\lambda=m+1$ & $m+1$ \\
$k \leq \kappa<k+1$ & $m<\lambda<m+1$ & $m+k+1$ \\
& between $m+k+1$ and $m+k+3$
\end{tabular}

In the third section we discuss the asymptotic behaviour of $f_{\kappa, \lambda}(z)$ as $z \rightarrow-\infty$. These formulas yield information on the values of $\kappa$ and $\lambda$ which produce a zero at $z=-\infty$. In the fourth section we shall give lower bounds for the number of zeros. Here functional equations will be derived to control the change of the number of zeros when $\kappa$ resp. $\lambda$ is replaced by $\kappa+1$ resp. $\lambda+1$. In the cases $\kappa \leq 0, \lambda \in \mathbb{R}$ or $\kappa>0$ and $\lambda \in \mathbb{N}$ we shall get the exact number of zeros. All these zeros are negative. The result is: $\left(k, m \in \mathbb{N}_{0}\right)$

\begin{tabular}{c|c|c}
\multicolumn{1}{c|}{$\kappa$} & $\lambda$ & $\begin{array}{c}\text { lower bounds for the number of zeros of } \\
f_{\kappa, \lambda}(z) \text { in } \mathbb{C}^{*}\end{array}$ \\
\hline \multicolumn{1}{c}{$\kappa \leq 0$} & $\lambda<0$ & 1 \\
$\kappa \leq 0$ & $m<\lambda \leq m+1$ & $m+1$ \\
$k<\kappa \leq k+1$ & $\lambda=m+1$ & $k+m+1$ \\
$k<\kappa \leq k+1$ & $m<\lambda<m+1$ & $m+1$
\end{tabular}

In the last case $(\lambda \notin \mathbb{N})$, we do not know the exact number of zeros. Some numerical calculations show that there may exist complex zeros.

1. Analytic continuation of $\boldsymbol{f}_{\kappa, \lambda}(z)$ in $\mathbb{C}^{*}$. In order to obtain the analytic continuation of $f_{\kappa, \lambda}(z)$ onto $\mathbb{C}^{*}$, we take the following integral representation of the moment sequences $(\kappa, \lambda<0)$

(1.1) $(n+1)^{\kappa} \log ^{\lambda}(n+1)$

$$
=\frac{1}{\Gamma(-\lambda)} \int_{0}^{1} t^{n} \int_{0}^{\infty} v^{-\lambda-1} \frac{(\log 1 / t)^{v-\kappa-1}}{\Gamma(v-\kappa)} d v d t \quad n=1,2, \ldots
$$

The equation is easily obtained by changing the integrals and using Euler's $\Gamma$-integral. (For the case $\kappa=0$, see Hardy [2], p. 268) The inner integral does 
not exist for $\lambda>0$. But partial integration furnishes a formula for $m \leq \lambda<$ $m+1\left(m \in \mathbb{N}_{0}\right), \kappa<0$ :

$$
\begin{aligned}
(n+1)^{\kappa} \log ^{\lambda}(n+1)= & \frac{(-1)^{m+1}}{\Gamma(m+1-\lambda)} \int_{0}^{1} t^{n} \int_{0}^{\infty} v^{m-\lambda}\left(\frac{d}{d v}\right)^{m+1} \\
& \times \frac{(\log 1 / t)^{v-\kappa-1}}{\Gamma(v-\kappa)} d v d t \quad n=0,1,2, \ldots
\end{aligned}
$$

This integral exists also for $n=0$ (for the existence see Lemma 2). For $\lambda=m$ the formula simplifies to

$$
\begin{aligned}
(n+1)^{\kappa} \log ^{m}(n+1)= & (-1)^{m} \int_{0}^{1} t^{n}\left(\log \frac{1}{t}\right)^{-\kappa-1} \sum_{\mu=0}^{m}\left(\begin{array}{l}
m \\
\mu
\end{array}\right)\left(\log _{2} \frac{1}{t}\right)^{\mu} \\
& \times\left(\frac{1}{\Gamma(-\kappa)}\right)^{(m-\mu)} d t \quad n=0,1,2, \ldots
\end{aligned}
$$

Using these integral representations of the coefficients, we get the following analytic continuation of $f_{\kappa, \lambda}(z)$ onto $\mathbb{C}^{*}$ :

a) $\kappa, \lambda<0$

$$
f_{\kappa, \lambda}(z)=\frac{z}{\Gamma(-\lambda)} \int_{0}^{1} \frac{t}{1-z t} \int_{0}^{\infty} v^{-\lambda-1} \frac{(\log 1 / t)^{v-\kappa-1}}{\Gamma(v-\kappa)} d v d t
$$

b) $\kappa<0, m \leq \lambda<m+1\left(m \in \mathbb{N}_{0}\right)$

$$
f_{\kappa, \lambda}(z)=\frac{(-1)^{m+1}}{\Gamma(m+1-\lambda)} \int_{0}^{1} \frac{1}{1-z t} \int_{0}^{\infty} v^{m-\lambda}\left(\frac{d}{d v}\right)^{m+1} \frac{(\log 1 / t)^{v-\kappa-1}}{\Gamma(v-\kappa)} d v d t
$$

In the special case $\lambda=m$, we have

$$
f_{\kappa, \lambda}(z)=(-1)^{m} \int_{0}^{1} \frac{1}{1-z t}\left(\log \frac{1}{t}\right)^{-\kappa-1} \sum_{\mu=0}^{m}\left(\begin{array}{l}
m \\
\mu
\end{array}\right)\left(\log _{2} \frac{1}{t}\right)^{\mu}\left(\frac{1}{\Gamma(-\kappa)}\right)^{(m-\mu)} d t
$$

c) In the case $k \leq \kappa<k+1 \quad\left(k \in \mathbb{N}_{0}\right), \lambda \in \mathbb{R}$, we consider the function $(1-z)^{k+1} f_{\kappa, \lambda}(z)$. It is easy to verify that the Taylor coefficients of this function at $z=0$ form a totally monotone sequence for $n \geq k+1$ and $\lambda<0$. Using similar methods as in a) and b) we obtain for example in case $k \leq \kappa<k+1, m<\lambda<m+1$

$$
\begin{aligned}
f_{\kappa, \lambda}(z) & =(1-z)^{-k-1}\left\{\sum_{n=0}^{k} z^{n} \sum_{\nu=0}^{n-1}\left(\begin{array}{c}
k+1 \\
\nu
\end{array}\right)(-1)^{\nu}(n+1-\nu)^{\kappa} \log ^{\lambda}(n+1-\nu)\right. \\
& \left.+\frac{z^{k+1}(-1)^{m+k}}{\Gamma(m+1-\lambda)} \int_{0}^{1} \frac{(1-t)^{k+1}}{1-z t} \int_{0}^{\infty} v^{m-\lambda}\left(\frac{d}{d v}\right)^{m+1} \frac{(\log 1 / t)^{v-\kappa-1}}{\Gamma(v-\kappa)} d v d t\right\}
\end{aligned}
$$

In case $\lambda=m$ the inner integral degenerates to a polynomial in the variable $\log _{2} 1 / t$. The degree of this polynomial is $m-1$ for integral $\kappa$ and $m$ 
otherwise. (Note that $1 / \Gamma(-k)=0$ for $k=0,1,2, \ldots$ ). In case $\kappa>0$ $z^{k} \sum_{\nu=0}^{k-1}\left(\begin{array}{c}k+1 \\ \nu\end{array}\right)(-1)^{\nu}(n+1-\nu)^{\kappa} \log ^{m}(n+1-\nu)$ can be represented by a similar integral and so we obtain

$$
\begin{aligned}
& f_{\kappa, m}(z)=(1-z)^{-k-1}\left\{\sum_{n=0}^{k-1} z^{n} \sum_{\nu=0}^{n-1}\left(\begin{array}{c}
k+1 \\
\nu
\end{array}\right)(-1)^{\nu}(n+1-\nu)^{\kappa} \log ^{m}(n+1-\nu)\right. \\
& \left.+z^{k}(-1)^{m+k+1} \int_{0}^{1} \frac{(1-t)^{k+1}}{(1-z t) t(\log 1 / t)^{\kappa+1}} \sum_{\mu=0}^{m}\left(\begin{array}{l}
m \\
\mu
\end{array}\right)\left(\log _{2} \frac{1}{t}\right)^{\mu}\left(\frac{1}{\Gamma(-\kappa)}\right)^{(m-\mu)} d t\right\}
\end{aligned}
$$

2. Upper bounds for the number of zeros. Using the formulas in $a$ ) $-c$ ) we can apply theorem 1 in [4]. Observe that the integrals in section 1 are of the form

$$
\int_{0}^{1} \frac{g_{\kappa, \lambda}^{\prime}(t)}{1-z t} d t
$$

In order to obtain an upper bound for the number of zeros of $f_{\kappa, \lambda}(z)$, we count the changes of sign of the functions $g_{\kappa, \lambda}^{\prime}(t)$ for $t \in(0,1)$. Obviously there are no changes of sign if $\kappa<0$ and $\lambda<0$ and at most $\lambda-1$ resp. $\lambda$ changes of sign if $\lambda \in \lambda \in \mathbb{N}$ and $\kappa>0$ and integral resp. $\kappa \in \mathbb{R}-\mathbb{N}$.

In the other cases we investigate the zeros of

$$
h_{\kappa, \lambda}(t)=\int_{0}^{\infty} v^{m-\lambda}\left(\frac{d}{d v}\right)^{m+1} \frac{(\log 1 / t)^{v}}{\Gamma(v-\kappa)} d v \text { for } t \in(0,1) \quad(m<\lambda<m+1) .
$$

Taking $a=\log _{2} 1 / t(a \in \mathbb{R})$ we obtain

$$
\left(\frac{d}{d a}\right)^{m+1} h_{\kappa, \lambda}\left(e^{-e^{a}}\right)=(\lambda-m) \cdots \lambda \cdot \int_{0}^{\infty} v^{m-\lambda} \frac{e^{a v}}{\Gamma(v-\kappa)} d v .
$$

If $\kappa<0$ then $\left(\frac{d}{d a}\right)^{m+1} h_{\kappa, \lambda}\left(e^{-e^{a}}\right)>0$ for $a \in \mathbb{R}$.

If $\kappa=0$ then $\left(\frac{d}{d a}\right)^{m} h_{\kappa, \lambda}\left(e^{-e^{a}}\right)>0$ for $a \in \mathbb{R}$.

Hence $h_{\kappa, \lambda}(t)$ has at most $(m+1)$ resp. $m$ zeros in $(0,1)$ according to the cases $\kappa<0$ resp. $\kappa=0$. In the case $\kappa>0$ a more careful investigation is necessary.

Lemma 1. Let $k, m \in \mathbb{N}_{0}$. If $m<\lambda<m+1$ and $k \leq \kappa<k+1$ then

has at most

$$
\Psi_{\kappa, \lambda}(t)=\frac{(-1)^{m+1}}{\Gamma(m+1-\lambda)} \int_{0}^{\infty} v^{m-\lambda}\left(\frac{d}{d v}\right)^{m+1} \frac{(\log 1 / t)^{v-\kappa-1}}{\Gamma(v-\kappa)} d v
$$

$$
\left\{\begin{array}{c}
m \\
m+2 \\
m+1
\end{array}\right\}
$$


zeros in $(0,1)$ if

$$
\left\{\begin{array}{c}
k \quad \text { is even and } \kappa-k+\lambda-m-1 \leq 0 \\
k \quad \text { is even and } \kappa-k+\lambda-m-1>0 \\
\text { otherwise }
\end{array}\right\}
$$

Proof. Let $b=\log 1 / t, b \in(0, \infty)$. Given $b>0$ we use Hankel's integral representation for $1 / \Gamma(z)$, i.e.

$$
\frac{1}{\Gamma(v-\kappa)}=\frac{1}{2 \pi i} \int_{C_{b}} e^{w} w^{\kappa-v} d w
$$

where the contour is chosen such that $|z| \geq b+\delta$ with $\delta>0$ for $z \in C_{b}$. Substituting this formula into (2.2) and changing the order of integration, we obtain

$$
\begin{aligned}
\Gamma(m+1-\lambda)( & -1)^{m+1} \Psi_{\kappa, \lambda}\left(e^{-b}\right) \\
& =\frac{b^{-\kappa-1}}{2 \pi i} \int_{C_{b}} e^{w} w^{\kappa} \int_{0}^{\infty} v^{m-\lambda}\left(\frac{d}{d v}\right)^{m+1}\left(\frac{b}{w}\right)^{v} d v d w \\
& =\frac{b^{-\kappa-1}}{2 \pi i} \int_{C_{b}} e^{w} w^{\kappa}\left(\log \frac{b}{w}\right)^{m+1} \int_{0}^{\infty} v^{m-\lambda} e^{-\log (w / b) v} d v d w \\
& =\frac{b^{-\kappa-1}}{2 \pi i} \int_{C_{b}} e^{w} w^{\kappa}(-1)^{m+1}\left(\log \frac{w}{b}\right)^{\lambda} \Gamma(m+1-\lambda) d w .
\end{aligned}
$$

Hence by moving the contour onto the real axis, we get

$$
\begin{aligned}
\Psi_{\kappa, \lambda}\left(e^{-b}\right)= & \frac{-b^{-\kappa-1}}{\pi}\left[\int_{-\infty}^{0} e^{w}|w|^{\kappa} \operatorname{Im}\left(e^{i \pi \kappa}\left(\log \frac{w}{b}+i \pi\right)^{\lambda}\right) d w\right. \\
& \left.\quad+\int_{0}^{b} e^{w} w^{\kappa} \operatorname{Im}\left\{\left(\log \frac{w}{b}\right)^{\lambda}\right\} d w\right] \\
= & \frac{b^{-\kappa-1}}{\pi}\left\{-\int_{0}^{\infty} e^{-w} w^{\kappa}\left|\log \frac{w}{b}+i \pi\right|^{\lambda} \sin \left(\pi \kappa+\lambda \arctan * \frac{\pi}{\log w / b}\right) d w\right. \\
= & \frac{1}{\pi}\left\{(-1)^{k+1} \int_{0}^{\infty} e^{-w b} w^{\kappa}|\log w+i \pi|^{\lambda} \sin (\pi(\kappa-k)\right. \\
& \left.\left.+\lambda \arctan \frac{\pi}{\log w}\right) d w+(-1)^{m+1} \sin \pi(\lambda-m) \int_{0}^{b} e^{w} e^{\kappa b}\left|\log \frac{w}{b}\right|^{\lambda} d w\right\} \\
& \left.=\frac{1}{\pi}\left(\log \frac{1}{w}\right)^{\lambda} d w\right\}
\end{aligned}
$$


with

$\arctan ^{*} \frac{\pi}{\log w}=\left\{\begin{array}{llll}\arctan \frac{\pi}{\log w}+\pi & \text { if } & 0<w \leq 1 & \\ \arctan \frac{\pi}{\log w} & \text { if } & w>1 & \text { (we use the main branch of }\end{array}\right.$

Therefore

$$
\begin{aligned}
(-1)^{m+1} \Phi_{2}^{(\nu)}(b) & >0 \text { for } b \in(0, \infty) \text { and } \\
\Phi_{1}^{(\nu)}(b) & \text { has at most as many zeros as the integrand (see [6], p. 50) } \\
& \text { for } \nu=0,1,2, \ldots
\end{aligned}
$$

Considering the behaviour of $\sin ($.$) and \arctan ($.$) , it is clear that the integrand$ of $\Phi_{1}(b)$ has $m$ zeros in $(0, \infty)$ if $\kappa-k+\lambda \leq m+1$ and $m+1$ zeros if $\kappa-k+\lambda>$ $m+1$. Since the derivatives have the same structure, the function $\left\{\Phi_{1}(b)+\right.$ $\left.\Phi_{2}(b)\right\}$ has at most as many zeros as $\Phi_{1}(b)$, if $\operatorname{sgn}\left(\Phi_{1}(b \rightarrow \infty)\right)=$ $\operatorname{sgn}\left(\Phi_{2}(b \rightarrow \infty)\right)=(-1)^{m+1}$ and at most one additional zero otherwise. The $\operatorname{sgn}\left(\Phi_{1}(b \rightarrow \infty)\right)$ is given by the sign of the integrand as $w \rightarrow 0$, i.e. the sign $\left((-1)^{k+1} \sin (\pi(\kappa-k)+\pi \cdot \lambda)\right)$. So we find the following cases: $\left(\nu \in \mathbb{N}_{0}\right)$

$k=2 \nu \quad \kappa-k+\lambda<m+1 \quad \Psi_{\kappa, \lambda}(t)$ has at most $m \quad$ zeros in $(0,1)$

$k=2 \nu \quad \kappa-k+\lambda>m+1 \quad \Psi_{\kappa, \lambda}(t)$ has at most $m+2$ zeros in $(0,1)$

$k=2 \nu+1 \quad \kappa-k+\lambda<m+1 \quad \Psi_{\kappa, \lambda}(t)$ has at most $m+1$ zeros in $(0,1)$

$k=2 \nu+1 \quad \kappa-k+\lambda>m+1 \quad \Psi_{\kappa, \lambda}(t)$ has at most $m+1$ zeros in $(0,1)$.

The following figures illustrate the situation:
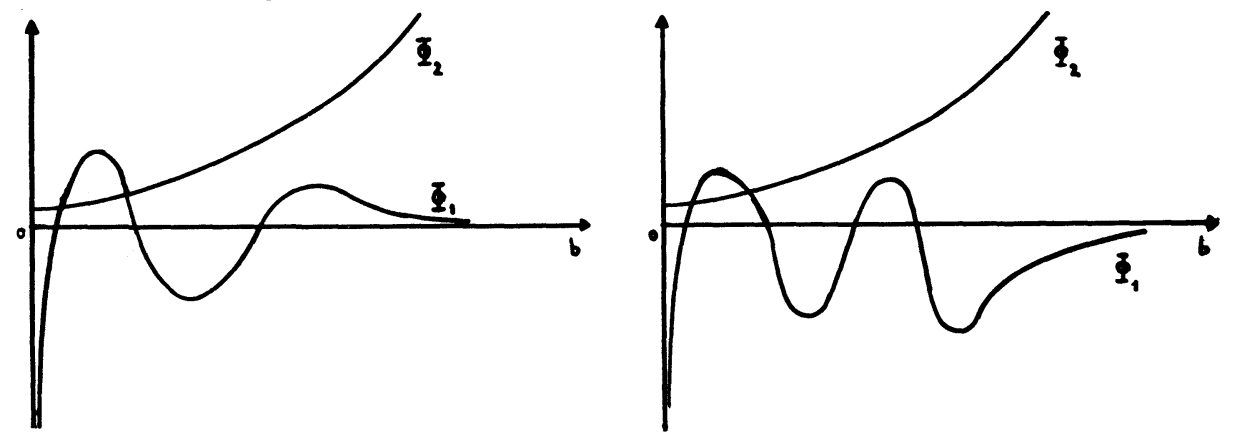

\begin{tabular}{|c|c|c|}
\hline$\kappa$ & $\lambda$ & upper bounds for the number of zeros of $f_{\kappa, \lambda}(z)$ in $\mathbb{C}^{*}$ \\
\hline $\begin{array}{c}\kappa \leq 0 \\
\kappa \leq 0 \\
k<\kappa \leq k+1 \\
k \leq \kappa<k+1\end{array}$ & $\begin{array}{r}\lambda<0 \\
m<\lambda \leq m+1 \\
\lambda=m+1 \\
m<\lambda<m+1\end{array}$ & $\begin{array}{cl} & \begin{array}{c}1(0 \\
m+1\end{array} \\
m+k+1 & \left.\text { if } \kappa^{2}+\lambda^{2}=0\right) \\
m+k+1 & \text { if } k \text { is even and } \kappa-k+\lambda-m-1 \leq 0 \\
m+k+3 & \text { if } k \text { is even and } \kappa-k+\lambda-m-1>0 \\
m+k+2 & \text { otherwise }\end{array}$ \\
\hline
\end{tabular}

The discussion in section 2 and theorem 1 in [4] yield the following result: $\left(k, m \in \mathbb{N}_{0}\right)$ 
REMARK. Using the same methods as in Lemma 1 and in No. 80 in [7, one can show that in case $\lambda<0$ and $k \leq k<k+1$ there are at most $k+2+\min \{k+1,[-\lambda]+2\}$ zeros in $\mathbb{C}^{*}$.

3. The asmptotic behaviour of $f_{\kappa, \lambda}(z)$ as $z \rightarrow-\infty$. In this section we investigate the asymptotic behaviour of

$$
\int_{0}^{1} \frac{g(t)}{1+x t} d t \text { as } x \rightarrow \infty .
$$

For $g(t)=g_{\kappa, \lambda}^{\prime}(t)$ (as treated at the beginning of section 2) the behaviour of (3.1) is essentially determined by the behaviour of $g_{\kappa, \lambda}^{\prime}(t)$ as $t \rightarrow 0$. Therefore we need the following

Lemma 2. Let $m, k \in \mathbb{N}_{0}$. Then

$$
\int_{0}^{\infty} v^{-\lambda-1} \frac{(\log 1 / t)^{v-\kappa}}{\Gamma(v-\kappa)} d v=\frac{1}{t}\left(\log \frac{1}{t}\right)^{-\lambda}(1+o(1)) \quad \text { for } \lambda<0 \text { and } \kappa \in \mathbb{R}
$$

$$
\begin{array}{r}
\int_{0}^{\infty} v^{m-\lambda}\left(\frac{d}{d v}\right)^{m+1} \frac{(\log 1 / t)^{v-\kappa}}{\Gamma(v-\kappa)} d v=(\lambda-m) \cdots \lambda \frac{1}{t}\left(\log \frac{1}{t}\right)^{-\lambda}(1+o(1)) \\
\text { for } m<\lambda<m+1, \kappa \in \mathbb{R}
\end{array}
$$

$$
\begin{aligned}
\int_{0}^{\infty} v^{m-\lambda} & \left(\frac{d}{d v}\right)^{m+1} \frac{(\log 1 / t)^{v-\kappa}}{\Gamma(v-\kappa)} d v=\left\{\frac{-1}{\Gamma(-\kappa)} \frac{\left.\log _{2} 1 / t\right)^{m}}{(\log 1 / t)^{\kappa}}\right. \\
& \left.-m\left(\frac{1}{\Gamma(-\kappa)}\right)^{\prime} \frac{\left(\log _{2} 1 / t\right)^{m-1}}{(\log 1 / t)^{\kappa}}\right\}(1+o(1)) \text { for } \lambda=m \quad \text { and } \quad \kappa \in \mathbb{R}
\end{aligned}
$$

for $t \rightarrow 0$.

\section{Proof.}

(3.2) Let $a=\log 1 / t, 0<\varepsilon<1 / 2$. Then

$$
\begin{aligned}
a^{-\lambda} \int_{0}^{\infty} v^{-\lambda-1} \frac{a^{v-\kappa}}{\Gamma(v-\kappa)} d v & =a^{-\lambda} \int_{a-a^{1-\varepsilon}}^{a+a^{1-\varepsilon}} v^{-\lambda-1} \frac{a^{v-\kappa}}{\Gamma(v-\kappa)} d v(1+o(1)) \\
& =\int_{a-a^{1-\varepsilon}}^{a+a^{1-\varepsilon}}\left(\frac{v}{a}\right)^{-\lambda} \frac{a^{v}}{\Gamma(v+1)} d v(1+o(1)) \\
& =\int_{a-a^{1-\varepsilon}}^{a+a^{1-\varepsilon}} \frac{a^{v}}{\Gamma(v+1)} d v(1+o(1)) \\
& =\sum_{a-a^{1-\varepsilon} \leq \nu \leq a+a^{1-\varepsilon}-1} \int_{\nu}^{\nu+1} \frac{a^{v}}{\Gamma(v+1)} d v(1+o(1)) \\
& =I(a)(1+o(1)) \text { for } a \rightarrow \infty
\end{aligned}
$$


Using the monotonicity of $a^{v} / \Gamma(v+1)$ for $1<v<a-1$ and $a<v<\infty$, we obtain

$$
\begin{aligned}
\sum_{a-a^{1-e} \leq \nu<a-2} \frac{a^{\nu}}{\nu !}+ & \sum_{a<\nu<a+a^{1-e}-1} \frac{a^{\nu+1}}{(\nu+1) !} \leq I(A) \\
& \leq \sum_{a-a^{1-e}<\nu<a-2} \frac{a^{\nu+1}}{(\nu+1) !}+\sum_{a<\nu<a+a^{1-\varepsilon}-1} \frac{a^{\nu}}{\nu !}+\int_{[a-2]+1}^{[a]+1} \frac{a^{v}}{\Gamma(v+1)} d v .
\end{aligned}
$$

Hence

$$
I(a)=\sum_{a-a^{1-\varepsilon} \leq \nu \leq a+a^{1-\varepsilon}} \frac{a^{\nu}}{\nu !}(1+o(1))=e^{a}(1+o(1)) \quad \text { for } \quad a \rightarrow \infty,
$$

which proves (3.2).

$$
\int_{0}^{\infty} \cdots d v=\int_{0}^{1} \cdots d v+\int_{1}^{\infty} \cdots d v=\mathrm{I}+\mathrm{II}
$$

It is clear that $\mathrm{I}=0$ (II) as $t \rightarrow 0$. After partial integration of II we apply (3.2) to obtain (3.3). (3.4) is trivial since the integral degenerates to a polynomial in the variable $\log _{2} 1 / t$.

Now we can derive the asymptotic behaviour of $f_{\kappa, \lambda}(z)$ for $z \rightarrow-\infty$.

Lemma 3. Let $\kappa \in \mathbb{R} ; k, m \in \mathbb{N}$. Then

$$
\begin{aligned}
& f_{\kappa, \lambda}(-x)=\frac{-1}{\Gamma(1-\lambda)}(\log x)^{-\lambda}(1+o(1)) \text { for } \quad \lambda<0 \\
& f_{\kappa, \lambda}(-x)=\frac{(-1)^{m+1}}{|\Gamma(1-\lambda)|}(\log x)^{-\lambda}(1+o(1)) \quad \text { for } \quad m<\lambda<m+1 \\
& f_{\kappa, \lambda}(-x)=\left\{\frac{(-1)^{m+k+1}}{|\Gamma(1-\kappa)|} \frac{\left(\log _{2} x\right)^{m+1}}{x(\log x)^{\kappa}}+\frac{m}{\kappa}(-1)^{m+k}\left|\left(\frac{1}{\Gamma(-\kappa)}\right)^{\prime}\right|\right. \\
& \left.\times \frac{\left(\log _{2} x\right)^{m}}{x(\log x)^{\kappa}}\right\}(1+o(1)) \quad \text { for } \quad \lambda=m+1, \quad k=[\kappa-] \quad \text { if } \quad \kappa>0 \\
& k=0 \quad \text { if } \quad \kappa<0 \\
& \kappa \neq 0
\end{aligned}
$$

$$
f_{\kappa, \lambda}(-x)=(-1)^{m+1} \frac{\left(\log _{2} x\right)^{m+1}}{x}(1+o(1)) \text { for } \lambda=m+1, \kappa=0
$$

for $x \rightarrow \infty$.

Proof. (3.5) Let $\kappa, \lambda<0$

$$
\begin{aligned}
f_{\kappa, \lambda}(-x) & =\frac{-x}{\Gamma(-\lambda)} \int_{0}^{1} \frac{t}{1+x t} \int_{0}^{\infty} v^{-\lambda-1} \frac{(\log 1 / t)^{v-\kappa-1}}{\Gamma(v-\kappa)} d v d t \\
& =\frac{-x}{\Gamma(-\lambda)} \int_{0}^{1 / \log x} \frac{t}{1+x t} \int_{0}^{\infty} v^{-\lambda-1} \frac{(\log 1 / t)^{v-\kappa-1}}{\Gamma(v-\kappa)} d v d t(1+o(1)) \quad \text { as } \quad x \rightarrow \infty
\end{aligned}
$$


1979]

ZEROS OF POWER SERIES

229

By (3.2) we find

$$
\begin{aligned}
f_{\kappa, \lambda}(-x)= & \frac{-1}{\Gamma(-\lambda)} x\left\{\int_{0}^{\log _{2} x} \frac{t}{1+x t} \frac{1}{t}\left(\log \frac{1}{t}\right)^{-\lambda-1} d t+\frac{1}{x} \int_{\log _{2} x / x}^{1 / \log x} \frac{1}{\frac{1}{x}+t}\right. \\
& \left.\times\left(\log \frac{1}{t}\right)^{-\lambda-1} d t\right\}(1+o(1)) \\
= & \left.O(x) t\left(\log \frac{1}{t}\right)^{-\lambda-1}\right|_{0} ^{\log _{2} x / x}+\frac{-1}{\Gamma(-\lambda)} \cdot \frac{x}{x} \int_{\log _{2} x / x}^{1 / \log x} \frac{1}{t} \\
& \times\left(\log \frac{1}{t}\right)^{-\lambda-1} d t(1+0(1)) \\
= & O\left(\log 2 x\left(\log x-\log _{3} x\right)^{-\lambda-1}\right)+\frac{-1}{\Gamma(-\lambda)} \cdot \frac{-1}{-\lambda}\left[\left(\log _{2} x\right)^{-\lambda}\right. \\
& \left.-(\log x)^{-\lambda}\left(1-\frac{\log _{3} x}{\log x}\right)^{-\lambda}\right](1+0(1)) \\
= & \frac{-1}{\Gamma(1-\lambda)}(\log x)^{-\lambda}(1+o(1)) \text { as } x \rightarrow \infty .
\end{aligned}
$$

(3.6) $k<\kappa<k+1, m<\lambda<m+1$. By (1.7) we know

$$
\begin{aligned}
& f_{\kappa, \lambda}(-x)=\frac{1}{(1+x)^{k+1}}\left\{P_{k}(-x)\right.+\frac{(-x)^{k+1}(-1)^{k+m}}{\Gamma(m+1-\lambda)} \\
&\left.\times \int_{0}^{1} \frac{(1-t)^{k+1}}{1+x t} \int_{0}^{\infty} v^{m-\lambda}\left(\frac{d}{d v}\right)^{m+1} \frac{\left(\log \frac{1}{t}\right)^{v-\kappa-1}}{\Gamma(v-\kappa)} d v d t\right\}
\end{aligned}
$$

$P_{k}(z)$ is a polynomial of degree $k$. For $x \rightarrow \infty$ we obtain by (3.3)

$$
\begin{aligned}
& f_{\kappa \cdot \lambda}(-x)=O\left(\frac{1}{x}\right)+(1+0(1)) \frac{(-1)^{m+1}}{\Gamma(m+1-\lambda)} \int_{0}^{1 / \log x} \frac{1}{1+x t}(\lambda-m) \cdot \cdots \cdot \lambda \\
& \times \frac{1}{t}\left(\log \frac{1}{t}\right)^{-\lambda-1} d t \\
& =O\left(\frac{1}{x}\right)+(1+o(1)) \frac{(-1)^{m+1}}{|\Gamma(-\lambda)|}\left\{\int_{0}^{1 / x \log _{2} x} \frac{1}{1+x t}\right. \\
& \left.\times \frac{1}{t}\left(\log \frac{1}{t}\right)^{-\lambda-1} d t+\frac{1}{x} \int_{1 / \times 2 \log _{2} x}^{1 / \log x} \frac{1}{\frac{1}{x}+t} \frac{1}{t}\left(\log \frac{1}{t}\right)^{-\lambda-1} d t\right\} \\
& =O\left(\frac{1}{x}\right)+(1+0(1)) \frac{(-1)^{m+1}}{|\Gamma(-\lambda)|}\left\{\int_{0}^{1 / x \log _{2} x} \frac{1}{t}\left(\log \frac{1}{t}\right)^{-\lambda-1} d t\right. \\
& \left.+O\left(\frac{1}{x}\right) \int_{1 / x \log _{2} x}^{1 / \log x} \frac{1}{t^{2}}\left(\log \frac{1}{t}\right)^{-\lambda-1} d t\right\}
\end{aligned}
$$

https://doi.org/10.4153/CMB-1979-029-9 Published online by Cambridge University Press 


$$
\begin{aligned}
= & O\left(\frac{1}{x}\right)+(1+o(1)) \frac{(-1)^{m+1}}{|\Gamma(-\lambda)|} \frac{-1}{-\lambda}(\log x)^{-\lambda}\left(1+\log _{3} x\right)^{-\lambda} \\
& +O\left(\frac{1}{x}\right)\left(-\log x\left(\log _{2} x\right)^{-\lambda-1}+x \log _{2} x\left(\log x+\log _{3} x\right)^{-\lambda-1}\right) \\
= & \frac{(-1)^{m+1}}{|\Gamma(1-\lambda)|}(\log x)^{-\lambda}(1+0(1)) .
\end{aligned}
$$

Similar calculations lead to the other cases.

Remark. It follows from (3.6) and (3.7) that for fixed $\lambda \in \mathbb{N}$, a zero is "generated" at $z=-\infty$, when $\kappa$ moves from $k-\varepsilon$ to $k+\varepsilon(k \in \mathbb{N})$.

4. Lower bounds for the number of zeros. The upper bounds for the number of zeros increase with $\kappa$ and $\lambda$. If $\kappa$ and $\lambda$ are both less than zero, then there exists exactly one zero, namely $z=0$. We look for methods which control the change of the number of zeros, when $\kappa$ or $\lambda$ increases, since the methods in [5], which give exactly the number of zeros in $\mathbb{C}^{*}$, fail in this situation. In the following we confine ourselves to the investigation of the real zeros. We proceed essentially as follows: If $\kappa$ is fixed and $\lambda$ increases we use the functional equation

$$
\frac{\partial}{\partial \kappa} f_{\kappa, \lambda}(z)=f_{\kappa, \lambda+1}(z)
$$

which follows directly from the power series representation. Using (4.1) we determine the sign of $f_{\kappa, \lambda+1}(z)$ at the negative zeros of $f_{\kappa, \lambda}(z)$ for $\kappa \leq 0$. We denote these zeros by $z_{i}=z_{i}(\kappa, \lambda)$. Now we need the following identity

$$
\begin{aligned}
\frac{\partial}{\partial \kappa} f_{\kappa, \lambda}\left(z_{i}\right) & =\sum_{n=1}^{\infty}(n+1)^{\kappa} \log ^{\lambda}(n+1) \cdot \log (n+1) z_{i}^{n} \\
& =\sum_{m=1}^{\infty}(n+1)^{\kappa}\left(\log ^{\lambda}(n+1)\right) \int_{0}^{1} \frac{1-t^{n}}{\log \frac{1}{t} d t z_{i}^{n}} \\
& =\int_{0}^{1} \frac{f_{\kappa, \lambda}\left(z_{i}\right)-f_{\kappa, \lambda}\left(z_{i} \cdot t\right)}{\log \frac{1}{t}} d t=\frac{1}{z_{i}} \int_{z_{i}}^{0} \frac{f_{\kappa, \lambda}(v)}{\log \frac{z_{i}}{v}} d v .
\end{aligned}
$$

The sign of $(\partial / \partial \kappa) f_{\kappa, \lambda}\left(z_{i}\right)$ is determined by the following Lemma.

Lemma 4. Given functions $f_{\nu}(x) \in C^{\nu}(-\infty, 0], \nu=0, \ldots, i-2(i \geq 2)$. Assume that each function $f_{\nu}(x)$ has at least $i-\nu$ zeros, that all zeros are simple and denote these zeros by

$$
0 \geq x_{1}(\nu)>x_{2}(\nu)>\cdots
$$


Suppose furthermore that

$$
\begin{gathered}
\left(x f_{\nu}(x)\right)^{\prime}=f_{\nu-1}(x) \text { in }(-\infty, 0] \\
f_{0}(0)=0 \text { and } f_{0}(x)<0 \text { for } x_{2}(0)<x<0,
\end{gathered}
$$

and that between two consecutive negative zeros of $f_{\nu-1}(x)$ there is exactly one zero of $f_{\nu}(x)$ for $\nu=1, \ldots, i-2$. Then

$$
\operatorname{sgn}\left[\frac{1}{x_{i}(0)} \int_{x_{i}(0)}^{0} \frac{f_{0}(t)}{\log \frac{x_{i}(0)}{t}} d t\right]=(-1)^{i}
$$

Proof. The idea of the proof is based on Wirsing's method [8], who proved the monotonicity of the zeros of $f_{\kappa, 0}(z)$ in $\kappa$. First observe that $f_{\nu}(0)=0$, i.e. $x_{1}(\nu)=0$, that

(4.4) $f_{\nu}^{\prime}(0)>0$ by (4.3) and that $f_{\nu}(x)<0$

$$
\text { in } x_{2}(\nu-1)<x<0 \text { for } \nu=1,2, \ldots, i-2 .
$$

Next our assumptions on the relative position of the zeros imply

$$
\begin{aligned}
& x_{k}(\nu)<x_{k-1}(\nu+1)<x_{k-1}(\nu) \text { for } \quad k=3,4, \ldots, i-\nu, \\
& \nu=0,1,2, \ldots, i-3 .
\end{aligned}
$$

Combining (4.5) with (4.4) and with the fact that all zeros are simple, we obtain (4.6) $\operatorname{sgn}\left(f_{\nu}(x)\right)=(-1)^{i-\nu-1}$ for $x_{i-\nu}(\nu)<x<x_{i-\nu-1}(\nu+1)$,

$$
\nu=0, \ldots, i-3
$$

By splitting the integral and partial integration we obtain

$$
\begin{aligned}
\frac{1}{x_{i}(0)} \int_{x_{i}(0)}^{0} \frac{f_{0}(t)}{\log \frac{x_{i}(0)}{t}} d t=\frac{1}{x_{i} 0} \int_{x_{i}(0)}^{x_{i-1}(1)} \frac{f_{0}(t)}{\log \frac{x_{i}}{t}} d t & \\
& \quad+\frac{t f_{1}(t)}{\left.\log \frac{x_{i}(0)}{t}\right|_{x_{i-1}(1)} ^{0}-\int_{x_{i-1}(1)}^{0} \frac{f_{1}(t)}{\left(\log \frac{x_{i}(0)}{t}\right)^{2}} d t}
\end{aligned}
$$

and after $(i-2)$ steps

$$
\frac{1}{x_{i}(0)} \int_{x_{i}(0)}^{0} \frac{f_{0}(t)}{\log \frac{x_{i}(0)}{t}} d t=\frac{1}{x_{i}(0)} \sum_{\nu=0}^{i-2} \nu !(-1)^{\nu} \int_{x_{i-\nu}(\nu)}^{x_{i-\nu-1}(\nu+1)} \frac{f_{\nu}(t)}{\left(\log \frac{x_{i}(0)}{t}\right)^{\nu+1}} d t
$$

(with $x_{1}(i-1)=0$ ). Our assertion follows from (4.4), (4.6) and $x_{i}(0)<0$.

Now we determine inductively the number of zeros of $f_{\kappa, \lambda}(z)$ for $m<\lambda \leq m+1$ $\left(m \in \mathbb{N}_{0}\right.$, in case $\lambda<0$ we have only one zero, namely $z=0$ ) in case $\kappa \leq 0$. We 
start with $f_{\kappa, \lambda-m}(z)$ which has exactly one zero $(z=0)$ for all $\kappa \leq 0$ (for this, observe the upper bound for the number of zeros). $f_{\kappa, \lambda-m+1}(z)$ has at least two zeros which follows from the asymptotic behaviour of this function (i.e. $f_{\kappa, \lambda-m+1}(x)>0$ for $\left.x \rightarrow-\infty, k \leq 0\right)$. The upper bound for the number of zeros shows that $f_{\kappa, \lambda-m+1}(z)$ has exactly two zeros for all $\kappa \leq 0$. For $i \geq 2$ and all $\kappa \leq 0$ we assume now, that $f_{\kappa, \lambda-m+i-1}(z)$ has exactly $i$ zeros, which are nonpositive and simple. Next we determine the $\operatorname{sgn}\left(f_{\kappa, \lambda-m+i}\left(z_{\mu}(\kappa, \lambda-m+i-\right.\right.$ 1))) for $\mu=2, \ldots, i$ with Lemma 4 , and the $\operatorname{sgn}\left(f_{\kappa, \lambda-m+i}(z)\right)$ as $z \rightarrow-\infty$ by the asymptotic behaviour for all $\kappa \leq 0$. To apply Lemma 4 we define $f_{\nu}(x)=$ $f_{\kappa-\nu, \lambda-m+i-1}(x)$ for $\nu=0, \ldots, \mu-2$. The assumptions of Lemma 4 are satisfied since we know the situation of $f_{\kappa, \lambda-m+i-1}(x)$ for $x \leq 0$ and all $\kappa \leq 0$. We find that $f_{\kappa, \lambda-m+i}(x)$ has $i$ changes of sign for $x<0$, hence $f_{\kappa, \lambda-m+i}(x)$ has at least $i+1$ nonpositive zeros for all $\kappa \leq 0$. The upper bound for the number of zeros shows that this is the exact number. Finally, we find for all $\kappa \leq 0$ that $f_{\kappa, \lambda}(z)$ has exactly $m+1$ zeros which are nonpositive and simple.

To investigate the cases $\kappa>0$, we use the functional equation

$$
\left(z f_{\kappa, \lambda}(z)\right)^{\prime}=f_{\kappa+1, \lambda}(z)
$$

which follows from the power series representation. If $k<\kappa \leq k+1, m<\lambda \leq$ $m+1\left(k, m \leq \mathbb{N}_{0}\right)$ we start with $f_{\kappa-(k+1), \lambda}(z)$ which has exactly $m+1$ zeros by the preceding discussion. Using (4.7) and Rolle's theorem we conclude that $f_{\kappa-k, \lambda}(z)$ has as many real zeros as $f_{\kappa-(k+1), \lambda}(z)$. If $z f_{\kappa-(k+1), \lambda}(z) \rightarrow 0$ as $z \rightarrow-\infty$, Rolle's theorem implies that $f_{\kappa-k, \lambda}(z)$ has at least one additional real zero. This condition is only satisfied for $f_{\kappa, \lambda}(z)$ if $\lambda \in \mathbb{N}_{0}$ and $\kappa>0$. So we get after $k+1$ steps that $f_{\kappa, \lambda}(z)$ has at least $m+1$ zeros if $\lambda \notin \mathbb{N}$ and $m+k+1$ zeros if $\lambda \in \mathbb{N}$. In case $k<\kappa \leq k+1, \lambda \in \mathbb{N}$, we could also find the exact number of zeros only using the first method (based on (4.1)): Given $k, m \in \mathbb{N}_{0}$, we have shown

\begin{tabular}{|c|c|c|c|c|}
\hline No & $\kappa$ & $\lambda$ & $\begin{array}{l}\text { lower bounds for the number } \\
\text { of zeros of } f_{\kappa, \lambda}(z) \text { in } \mathbb{C}^{*}\end{array}$ & Remarks \\
\hline i) & $\kappa \leq 0$ & $\lambda \leq 0$ & $1\left(\right.$ resp. 0 if $\left.\kappa^{2}+\lambda^{2}=0\right)$ & $z=0$ \\
\hline ii) & $\kappa \leq 0$ & $m<1 \leq m+1$ & $m+1$ & all zeros (except $z=0$ ) \\
\hline iii) & $k<\kappa \leq k+1$ & $\lambda=m+1$ & $m+k+1$ & negative and \\
\hline iv) & $k<\kappa \leq k+1$ & $m<\lambda<m+1$ & $m+1$ & increasing with $\kappa$ \\
\hline
\end{tabular}

Remarks. 1) In the proceeding proof it was essential to make an induction first with respect to $\lambda$ (based on (4.1)) and then with respect to $\kappa$ (based on (4.7)), since otherwise Lemma 4 would not be applicable since we do not get the exact number of zeros in this case.

2) The monotonicity of the negative zeros in $\kappa$ follows from the equation

$$
\left.0=\frac{d}{d \kappa} f_{\kappa, \lambda}\left(z_{i}(\kappa, \lambda)\right)=\frac{\partial}{\partial \kappa} f_{\kappa, \lambda}(\kappa, \lambda)\right)+\frac{d}{d \kappa} z_{i}(\kappa, \lambda) f_{\kappa, \lambda}^{\prime}\left(z_{i}(\kappa, \lambda)\right)
$$

and Lemma 4. 
3) As already mentioned in (1) the number of zeros in case (iv) is not the exact number in general, which follows immediately from case (iii). In case (iv) also complex zeros occur, as it is shown by numerical calculations for the following case: A zero of $f_{5,1}(z)$ lies in a neighbourhood of $z=0,54507+i \cdot 0,44807$. Other calculations showed that one might expect complex zeros in case (iv) in general.

\section{BIBLIOGRAPHY}

1. D. Borwein and W. Kratz, On the zeros of the power series $\sum_{n=0}^{\infty}(-1)^{n}\left(1-c^{-n-1}\right)^{\kappa} z^{n}$ with an application to discontinuous Riesz-summability. Canadian Math. Bull., Vol. 19 (4), (1976), 417-424.

2. G. H. Hardy, Divergent series. Clarendon Press, Oxford 1948.

3. W. B. Jurkat and A. Peyerimhoff, On power series with negative zeros. Tôhoku Math. J. II, Vol. 24 (1972), 207-221.

4. W. Gawronski and A. Peyerimhoff, On the zeros of power series with rational coefficients. Archiv für Mathematik, Vol. 29, Fasc. 2 (1977), 173-186.

5. A. Peyerimhoff, On the zeros of power series. Mich. Math. J. 13 (1966), 193-214.

6. A. Peyerimhoff, Lectures on summability. Lecture Notes in Math. 107, Springer Verlag 1968.

7. Polyá-Szegö, Aufgaben aund Lehrsätze aus der Analysis. Springer Verlag, HT Bd. 74, 1971.

8. E. Wirsing, On the monotonicity of the zeros of two power series. Mich. Math. J. 13 (1966), 215-218.

\section{ABTEILUNG FÜr MATHEMATIK I \\ UNIVERSTTÄT ULM \\ 7900 Ulm WeSt GERMANY}

